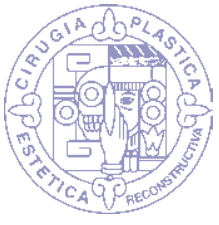

doi: 10.35366/98341

\section{Lipoma tenar gigante causa inusual de síndrome del túnel del carpo}

\author{
Giant palmar lipoma an unusual cause of carpal tunnel syndrome \\ Dr. Israel Salazar-Vizuet,* Dra. Mónica Gisela Cobos-Bonilla, \\ Dra. Andrea del Villar-Trujillo, ${ }^{*}$ Dr. Fernando Romero-Espinosa ${ }^{\S}$
}

\footnotetext{
Palabras clave: Lipoma, túnel del carpo, compresión nerviosa.

Keywords: Lipoma, carpal tunnel, nerve compression.
}

\footnotetext{
${ }^{*}$ Médico adscrito de base.

‡ Médico residente de Cirugía Plástica y Reconstructiva.

$\S$ Jefe de Servicio de Cirugía Plástica y Reconstructiva.

Departamento de Cirugía Plástica y Reconstructiva, Centro Médico «Lic. Adolfo López Mateos».

Recibido:

18 diciembre 2019

Aceptado:

14 septiembre 2020
}

\section{RESUMEN}

Describimos la presencia de síndrome del túnel del carpo secundario a lipoma gigante en una mujer de 53 años, valorada en la consulta externa de cirugía plástica y reconstructiva. Se inició protocolo de estudio y se ofreció manejo quirúrgico, se obtuvo una mejoría clínica del $100 \%$ a las cuatro semanas de la cirugía. No presentó recidiva a los seis meses postquirúrgicos. Fue un hallazgo interesante de etiología poco común como causa de una entidad clínica muy frecuente. Esto debe recodarnos que en el diagnóstico diferencial de un tumor en la mano se debe considerar un lipoma.

\section{ABSTRACT}

We describe the presence of carpal tunnel syndrome secondary to a giant lipoma in a 53-year-old woman, assessed in the plastic and reconstructive surgery outpatient clinic. A study protocol was begun and surgical management was offered, attaining a $100 \%$ clinical improvement, four weeks after surgery. Six months after surgery she did not have a recurrence. It was an interesting finding with a rare etiology as a cause of a very frequent clinical entity. This should remind us that in the differential diagnosis of a tumor in the hand, a lipoma should be considered.

\section{INTRODUCCIÓN}

L os lipomas son tumores benignos de tejidos ـ blandos muy frecuentes. ${ }^{1}$ Son los tumores más frecuentes en adultos, con una prevalencia en la población general de 1\%, ${ }^{2,3}$ más comunes en individuos obesos. ${ }^{4,5}$ Estas neoplasias benignas de tejidos blandos se desarrollan típicamente en la quinta a séptima década de la vida. ${ }^{4,6}$ Los lipomas rara vez se encuentran en niños. ${ }^{4,7}$ Se pueden situar en cualquier parte del cuerpo, pero su presencia en la mano es rara. Dieciséis por ciento de los tumores blandos de la mano son lipomas y se localizan, de manera predominante, en la musculatura tenar e hipotenar. $^{2,8}$ Los lipomas se consideran tumores gigantes cuando tienen un diámetro de $5 \mathrm{~cm} \mathrm{o}$ más, por lo general se comportan como tumo- raciones asintomáticas, de crecimiento lento y progresivo. Sin embargo, algunas veces pueden provocar síntomas debido a la compresión de estructuras adyacentes. ${ }^{1,9}$

La compresión del nervio periférico por lipoma es poco frecuente. En la literatura se describen pocos casos de síndrome del túnel del carpo como resultado de la compresión del nervio mediano por un lipoma. ${ }^{10,11}$

\section{CASO CLÍNICO}

Paciente femenino de 53 años con presencia de masa tumoral de crecimiento lento de un año de evolución en la superficie tenar de la mano izquierda. A los seis meses presentó alteraciones de la flexión, seguida de la pérdida de fuerza del primer y segundo dedo, con caída

Citar como: Salazar-Vizuet I, Cobos-Bonilla MG, del Villar-Trujillo A, Romero-Espinosa F. Lipoma tenar gigante causa inusual de síndrome del túnel del carpo. Cir Plast. 2020; 30 (3): 160-166. https://dx.doi.org/10.35366/98341 
de objetos de la mano, así como presencia de parestesias en territorio del nervio mediano, las cuales eran incapacitantes para realizar sus actividades diarias, motivo por el que acudió con médico familiar, quien la refirió al Servicio de Cirugía Plástica y Reconstructiva.

En su valoración se encontró a la exploración de la mano izquierda una tumoración en la eminencia tenar, indolora, de consistencia firme, flexible y relativamente móvil, no adherida a planos profundos. Se realizó transiluminación en la tumoración y fue negativa. No tenía atrofia en la musculatura tenar (Figura 1). A la exploración dirigida se encontró signos de Tinel y Phalen positivos. Se inició protocolo prequirúrgico y se solicitó imagen por resonancia magnética, encontrando en fase $\mathrm{T} 2$ imagen homogénea con aumento de intensidad, que reportó probable lipoma en eminencia tenar de 54.98 por $51.88 \mathrm{~mm}$ (Figura 2).

Se realizó electromiografía de la muñeca, la cual identificó aspectos compatibles con lesión marcada del nervio mediano izquierdo. Se decidió efectuar manejo quirúrgico por compresión extrínseca del nervio mediano. Se realizó resección quirúrgica de la tumoración de la eminencia tenar bajo sedación y bloqueo regional con isquemia, encontrando lesión superficial, solitaria, de bordes bien delimitados, redonda, movible, de consistencia blanda, color amarillo, con aspecto homogéneo semejante a tejido adiposo (Figura 3), con una delgada cápsula y medidas de $5 \times 5.2 \mathrm{~cm}$ (Figura 4). Al realizar la exéresis de la lesión se observó integridad del flexor digitorum y ramas terminales del nervio mediano (Figura 5). Se envió a estudio histopatológico, mismo que reportó lipoma de patrón clásico (Figura 6).

\section{Revisión bibliográfica}

Los lipomas son muy comunes y se encuentran entre las neoplasias de tejidos blandos más ampliamente distribuidas del cuerpo. ${ }^{12}$ Se presentan en varias ubicaciones anatómicas dentro de la palma. Los lipomas superficiales surgen en los tejidos subcutáneos. Los lipomas profundos surgen en el canal de Guyon, el túnel carpiano y el espacio palmar profundo. Entre las series más grandes que diferencian los lipomas superficiales y palmares profundos están las de Booher. ${ }^{13}$
Histológicamente, los lipomas son casi indistinguibles del tejido adiposo normal, ${ }^{4}$ están compuestos principalmente de adipocitos maduros; estas lesiones son uniformes en forma y tamaño y están bien circunscritas. Una cápsula rodea habitualmente una masa lobular suave, de color amarillo a naranja. Aunque el aspecto histológico se parece al tejido adiposo maduro, los lipomas no derivan de adipocitos maduros, sino de preadipocitos mesenquimatosos. ${ }^{6}$

Su etiología es desconocida, se han propuesto múltiples factores causales que incluyen factores genéticos, ${ }^{14}$ traumáticos ${ }^{15,16}$ y metabólicos. ${ }^{17,18}$ La principal teoría genética para la formación de lipomas propone que las anomalías cariotípicas espontáneas conducen a productos de fusión cromosómica que promueven la proliferación de adipocitos. ${ }^{14}$ Aunque se han descrito muchas otras alteraciones cromosómicas, los lipomas se asocian más comúnmente con traslocaciones y reordenamientos de la región cromosómica 12q13-q15. ${ }^{14,19}$

La formación de un lipoma después de un trauma físico ha sido reportada por múltiples investigadores. Durante décadas se especuló que los tumores lipomatosos que surgieron después de un traumatismo no eran en realidad una proliferación de adipocitos, sino una herniación de tejido adiposo preexistente a través de la fascia suprayacente. ${ }^{20,21}$ Estas lesiones no encapsuladas se denominaron «pseudolipomas».

Los lipomas se han asociado con numerosos procesos fisiopatológicos. Se han observado en pacientes con diabetes, ${ }^{17}$ hiperlipidemia, ${ }^{4}$ disfunción mitocondrial, ${ }^{22}$ endocrinopatías

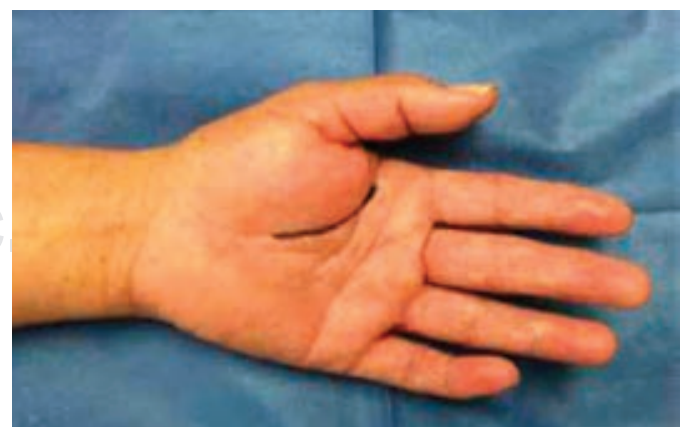

Figura 1: Lesión en la eminencia tenar de la mano izquierda. 


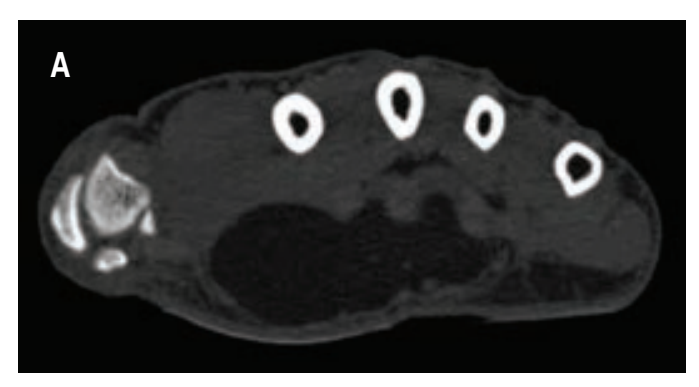

Figura 2:
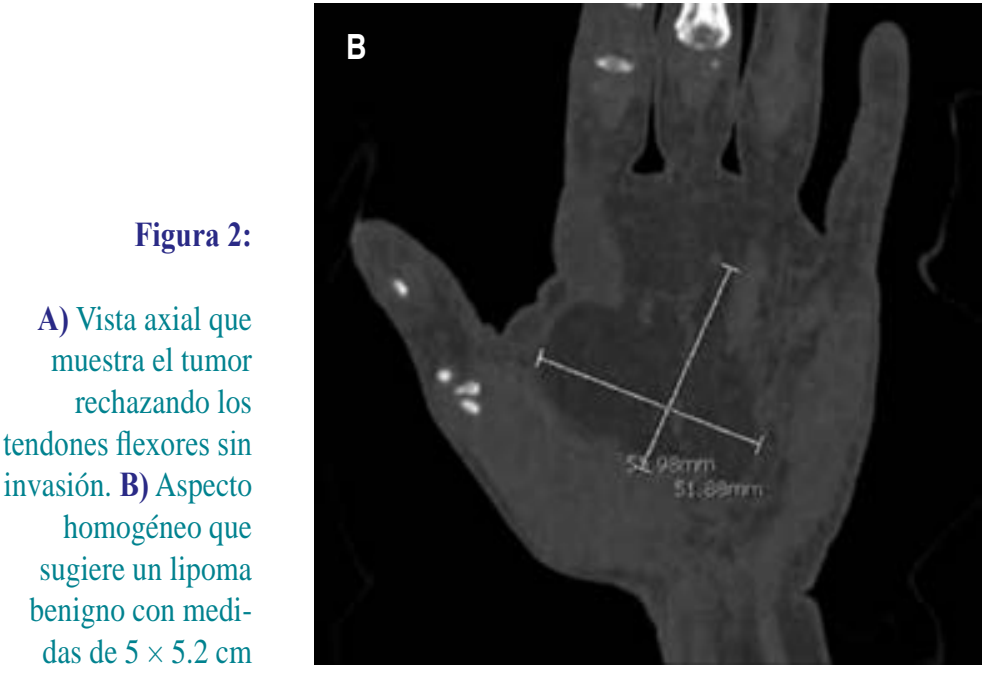

como el bocio nodular, ${ }^{23}$ neoplasias endocrinas múltiples tipo $1^{24}$ y síndrome de Cushing. ${ }^{12}$ También se informó un caso de desarrollo de lipoma en un paciente diabético tratado con clorpropamida. ${ }^{25}$ Estos hallazgos sugieren un papel para la disfunción metabólica en el desarrollo de neoplasias lipomatosas.

Aunque histológicamente son benignos y por lo general asintomáticos, se ha informado que los lipomas causan síntomas en la extremidad superior debido a la compresión de las estructuras neurovasculares y la interferencia con la función articular y tendinosa. ${ }^{7}$ Asimismo, se han notificado neuropatías por compresión del nervio interóseo posterior, ${ }^{7,12,26}$ nervio ulnar, ${ }^{27,28}$ nervio radial superficial, «nervios digitales» y el nervio mediano en el canal carpiano. 7,29,30

Por lo regular, se comportan como tumoraciones asintomáticas de crecimiento lento y progresivo; sin embargo, algunas veces pueden conducir a sintomatología debido a la compresión de estructuras adyacentes. Los lipomas se consideran tumores gigantes cuando tienen 5 cm o más de diámetro. ${ }^{1,9}$ La mayoría de las veces los lipomas de la mano se presentan como una masa solitaria, son asintomáticos y sólo reciben atención clínica cuando son de interés estético o se vuelven lo suficientemente grandes como para causar deterioro mecánico. La compresión del nervio periférico por lipoma es muy poco frecuente. En la literatura se describen pocos casos de síndrome del túnel carpiano como resultado de la compresión del nervio mediano por un lipoma. ${ }^{10,11}$

Rodríguez ${ }^{31}$ informó sobre 13 lipomas de la mano y muñeca, seis de los cuales causaron compresión nerviosa y de éstos sólo uno causó síntomas del nervio mediano. Leffert ${ }^{29}$ informó una serie de 141 lipomas de la extremidad superior observados durante un periodo de 10 años: 109 tumores eran asintomáticos y se extirparon únicamente por razones estéticas. De las 32 lesiones sintomáticas, 26 causaron dolor o sensibilidad y seis produjeron parestesias o déficit sensorial secundario a la compresión nerviosa. Paarlberg ${ }^{32}$ y Linscheid informaron que 59 pacientes recolectados durante un periodo de 25 años tenían lipomas de la mano, de éstos, tres tenían síntomas de compresión nerviosa y dos eran del nervio mediano en el canal carpiano.

La historia y el examen físico son la base del diagnóstico. Los lipomas se presentan con mayor frecuencia como una masa no densa, blanda y móvil de crecimiento lento. Cuando es subcutáneo, el diagnóstico se puede hacer mediante una sensación de «masa» característica en la palpación. La aplicación de una bolsa

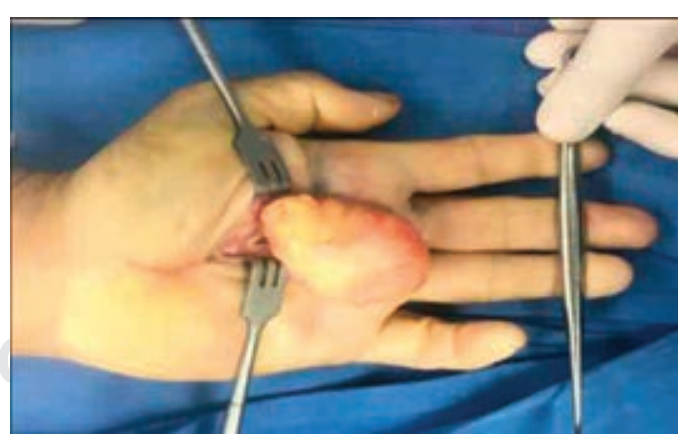

Figura 3: Imagen intraoperatoria en la que se observa lesión superficial solitaria de bordes bien delimitados, redonda, móvil y de consistencia blanda, de color amarillo con aspecto homogéneo, que se asemeja a tejido adiposo. 

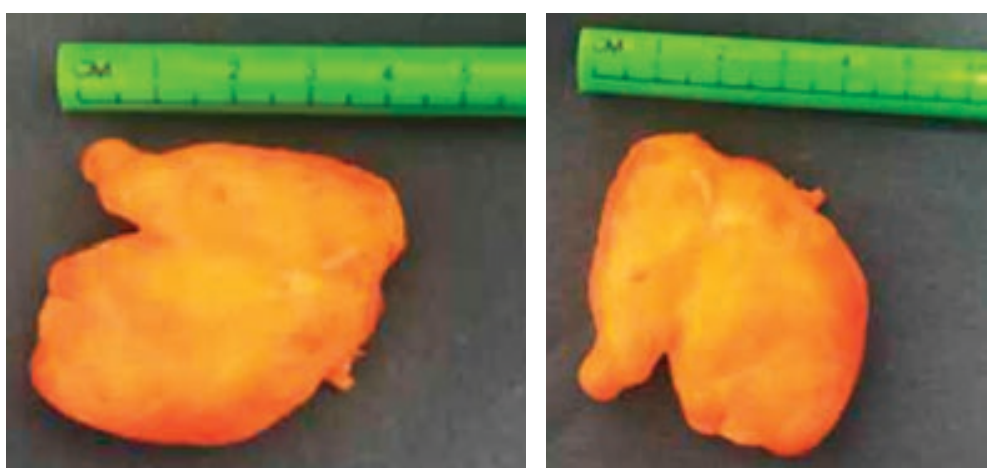

Figura 4: Tumor con medidas de $5 \times 5.2 \mathrm{~cm}$.

de hielo al tumor para enfriar y endurecer la grasa también se ha utilizado para ayudar en el diagnóstico. ${ }^{33}$ En ocasiones, los lipomas de la mano pueden ser difíciles de diferenciar de los quistes ganglionares por palpación. Los quistes ganglionares permiten el paso de la luz en la transiluminación, mientras que los lipomas no lo hacen. ${ }^{2}$ En pacientes que presentan síntomas de neuropatía por compresión, un signo positivo de Tinel (parestesia distal secundaria a la percusión sobre el tumor) puede ser útil para confirmar que un lipoma es la fuente de compresión nerviosa. ${ }^{34}$

Cuando un tumor subcutáneo no puede ser diagnosticado por palpación, o cuando un lipoma se desarrolla dentro de estructuras más profundas donde la palpación es difícil, la imagen puede ser necesaria para el diagnóstico. En una radiografía simple los lipomas aparecen como un área radiolúcida característica denominada «densidad de agua clara». ${ }^{29}$ La ecografía muestra un área hiperecoica homogénea y circunscrita. ${ }^{35,36}$ Con la tomografía computarizada (TC) los lipomas exhiben bordes suaves con márgenes definidos, ${ }^{37}$ una densidad uniforme comparable a la grasa normal ${ }^{38,39}$ y no mejoran con el contraste intravenoso. ${ }^{37}$ El uso de imágenes por resonancia magnética (RM) es homogéneo, se puede observar una señal de alta intensidad similar a la grasa subcutánea. ${ }^{38,39}$ La resonancia magnética es preferible, ya que es altamente sensible y específica para el diagnóstico. En general, se recomienda una RM para la evaluación de todas las masas lipomatosas subfasciales de la extremidad superior. Para los lipomas subcutáneos la RM está indicada sólo para tumores de $5 \mathrm{~cm}$ o más de diámetro. ${ }^{3,8}$ Cualquier tumoración de tejido blando que sea mayor de $5 \mathrm{~cm}$ debe considerarse maligno hasta que se demuestre lo contrario.

Chung y su equipo ${ }^{40}$ estudiaron las características de la RM para diferenciar los tumores benignos de los tejidos blandos malignos (incluidas las masas lipomatosas) de las extremidades. Mostraron que tanto las lesiones benignas como las malignas pueden tener las mismas características de la RM, aunque un diámetro de $5 \mathrm{~cm}$ o más y la heterogeneidad tumoral son dos características que se observan con mayor frecuencia en las lesiones malignas. ${ }^{40}$

En cuanto al tratamiento, las lesiones asintomáticas pequeñas que han sido diagnosticadas por la historia y el examen físico pueden observarse sin intervención. Sin embargo, la escisión quirúrgica está indicada cuando hay dolor, interferencia con la función de la mano, neuropatía por compresión o preocupación estética. ${ }^{29,41}$

En raras ocasiones, en pacientes con síndrome del túnel carpiano, la infiltración extensa de nervios por un lipoma puede no permitir la resección del tumor sin causar daño neurológico permanente. Para tales presentaciones complejas, la división del retináculo flexor sin
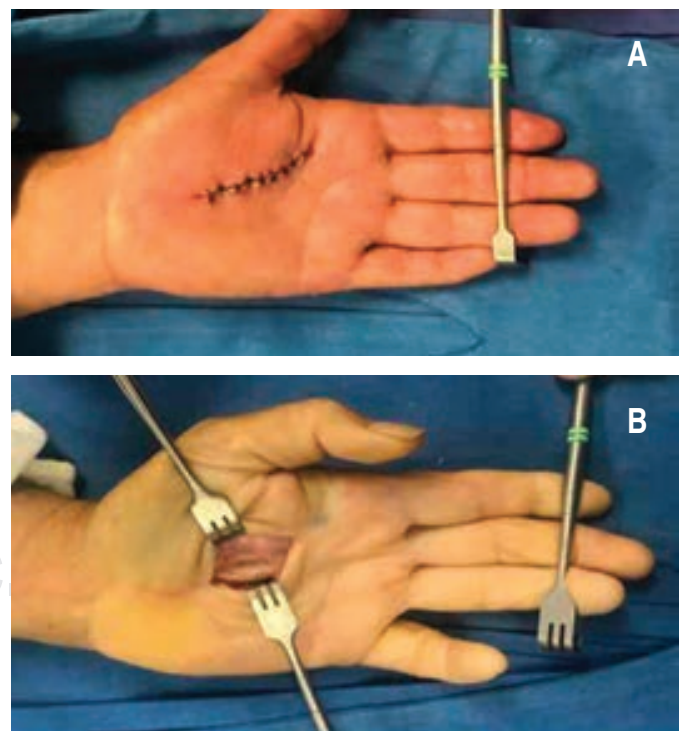

Figura 5: A) Integridad del flexor digitorum y ramas terminales del nervio mediano. B) Postquirúrgico inmediato. 

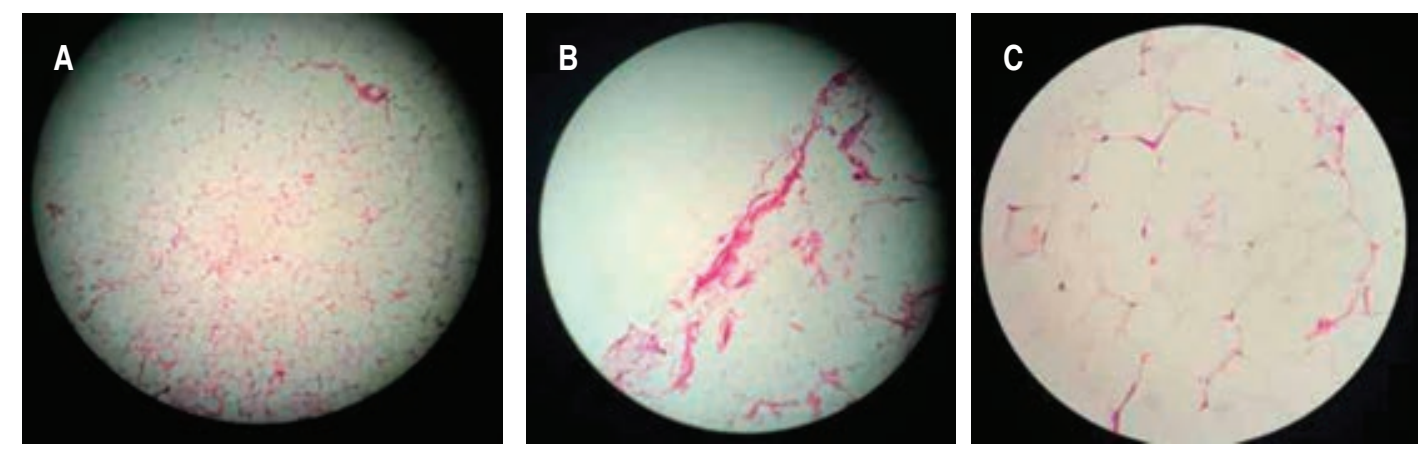

Figura 6: A) Periferia del tumor, cápsula delgada de tejido conectivo que delimita al tejido adiposo maduro. B) Tumor formado por tejido adiposo maduro sin atipia celular ni mitosis, creando lóbulos separados por tejido fibroconectivo. C) Las células son adipocitos maduros sin atipia nuclear ni celular.

escisión del tumor es una buena estrategia para el alivio de los síntomas. ${ }^{42}$ Los métodos alternativos de extirpación del tumor, como la liposucción ${ }^{43}$ y la escisión asistida endoscópicamente, ${ }^{44}$ tienen como objetivo minimizar la cicatrización. Además, se han utilizado inyecciones de mesoterapia o fosfatidilcolina ${ }^{45} y$ desoxicolato ${ }^{46}$ intralesionales para reducir los lipomas pequeños. Sin embargo, la resección quirúrgica u observación sin intervención sigue siendo el estándar de atención.

\section{DISCUSIÓN}

Los lipomas consisten en células grasas maduras que pueden aparecer en una localización subcutánea, intramuscular o intermuscular. La recurrencia local es infrecuente después de la escisión marginal. Los lipomas representan $16 \%$ de todos los tumores mesenquimales ${ }^{46,47}$ y con frecuencia son el primer tipo de tumor benigno en las extremidades. Aunque están localizados predominantemente en las extremidades inferiores, su localización en la mano es particularmente interesante debido a su expresión atípica.

Myhre-Jensen, ${ }^{48}$ en una revisión clínica de 1,331 tumores blandos benignos que incluyen 640 lipomas, encontró un tamaño de tumor menor a $5 \mathrm{~cm}$, en el $95 \%$ de los casos. Al mismo tiempo, más de $50 \%$ de los 72 sarcomas de tejidos blandos malignos diagnosticados durante el estudio midieron $5 \mathrm{~cm}$ o más.

La RM es la modalidad de visualización de elección para la exploración de tumores de la mano. Principalmente permite una orientación diagnóstica del tumor y, en segundo lugar, muestra relaciones anatómicas entre el tumor y las estructuras neurovasculares. Clásicamente los lipomas aparecen como una masa homogénea con un borde afilado, hipersignales T1 y T2 espontáneos, intensidad de señal reducida después del borrado de la señal de grasa, sin aumento en la señal con el agente de contraste de gadolinio. Los cortes finos menores de $2 \mathrm{~mm}$ permiten la identificación de septos dentro del tumor, que aparecen como rasgos pequeños y discretamente elevados después de la inyección de gadolinio. ${ }^{48,49}$

Es evidente a partir del tiempo necesario para recopilar los casos de la serie mencionada anteriormente, que los lipomas de la mano y la muñeca no son comunes y los lipomas que causan la compresión del nervio mediano aún menos.

En nuestro caso, la paciente acudió por incapacidad para realizar sus actividades diarias. No tenía comorbilidades o traumatismo previo, con presencia de túnel del carpo que ameritó tratamiento quirúrgico, con recuperación de sus actividades diarias, sin recurrencias al momento.

No debemos subestimar esta patología, ya que con frecuencia puede ser asintomática, puede llegar a ser incapacitante y si no se realiza un diagnóstico y tratamiento adecuado, puede llegar a causar pérdida de la pinza básica y atrofia importante en el territorio del nervio mediano. 


\section{CONCLUSIONES}

Reportamos este caso como un hallazgo interesante de una etiología inusual como causa de una entidad clínica muy común: el síndrome del túnel carpiano. Esto debe recordarnos como profesionales de la salud que en el diagnóstico diferencial de la masa del tejido blando en la mano y la muñeca se debe considerar un lipoma, cuya presentación clínica varía desde asintomática hasta la incapacidad por la compresión nerviosa.

\section{REFERENCIAS}

1. Nadar MM, Bartoli CR, Kasdan ML. Lipomas of the hand: a review and 13 patient case series. Eplasty 2010; 10: e66.

2. Ingari JV, Faillace JJ. Benign tumors of fibrous tissue and adipose tissue in the hand. Hand Clin 2004; 20 (3): 243.

3. Al-Qattan MM, Al-Lazzam AM, Al Thunayan A, Al Namlah A, Mahmoud S, Hashem F et al. Classification of benign fatty tumors of the upper limb. Hand Surg 2005; 10(1):43.

4. Weiss SW, Goldblum JR. Benign lipomatous tumors. In: Enzinger FM, Weiss SW, eds. Soft tissue tumors. 3rd ed. St Louis, Mo: Mosby 1995, pp. 381-430.

5. Froimson Al. Benign solid tumors. Hand Clin 1987; 3 (2): 213-217.

6. Phalen GS, Kendrick JI, Rodriguez JM. Lipomas of the upper extremity: a series of fifteen tumors in the hand and wrist and six tumors causing nerve compression. Am J Surg 1971; 121 (3): 298-306.

7. White W. Hanna D. Troublesome lipomata of the upper extremity. J Bone Joint Surg 1962; 44A: 1353-1359.

8. Cribb GL, Cool WP, Ford DJ, Mangham DC. Giant lipomatous tumors of the hand and forearm. J Hand Surg Br 2005; 30B: 509.

9. Fazilleau F, Williams T, Richou J, Sauleau V, Nen D. Median nerve compression in carpal tunnel caused by a giant lipoma. Case Reports in Orthopedic 2014 (2): 654934. doi: 10.1155/214/654934.

10. Azwa N, Shalimar A, Jamari S. A palmar lipoma with median and ulnar nerve compressive symptoms. Malays Orthop J 2007; 1: 33-55.

11. Sbai MA, Benzarti S, Msek H, Boussen M, Khorbi A. Carpal tunnel syndrome caused by lipoma: a case report. Pan Afr Med J 2015; 22: 51-57.

12. Barber K, Bianco A. Soule E, McCarty C. Benign extraneural soft tissue tumors of the extremities causing compression of nerves. J Bone Joint Surg 1962; 44A: 98.

13. Booher RJ. Lipoblastic Tumors of the hands and feet review of the literature and report of thirty-three cases. J Bone Joint Surg 1965; 47 (4): 727-740.

14. Sandberg AA. Updates on the cytogenetics and molecular genetics of bone and soft tissue tumors: lipoma. Cancer Genet Cytogenet 2004; 150 (2): 93-115.

15. Signorini M, Campiglio GL. Posttraumatic lipomas: where do they really come from? Plast Reconstr Surg 1998; 101 (3): 699-705.
16. Copcu E, Sivrioglu NS. Posttraumatic lipoma: analysis of 10 cases and explanation of possible mechanisms. Dermatol Surg 2003; 29 (3): 215-220.

17. Weinberg T, Feldman M, Sr. Lipomas of the gastrointestinal tract. Am J Clin Pathol 1955; 25 (3): 272-281.

18. Miyake O, Hara T, Matsumiya K, Oka T, Takaha $M$, Kurata A. Adrenal myelolipoma associated with Cushing's syndrome: a case report. Hinyokika Kiyo 1992; 38 (6): 681-684.

19. Sandberg AA, Bridge JA. Tumors of Fat. The cytogenetics of bone and soft tissue tumors. Austin: RG Landes Company 1994:147-192.

20. Brooke RI, MacGregor AJ. Traumatic pseudolipoma of the buccal mucosa. Oral Surg Oral Med Oral Pathol 1969; 28 (2): 223-225.

21. Aust MC, Spies M, Kall S, Jokuszies A, Gohritz A, Vogt P. Posttraumatic lipoma: fact or fiction? Skinmed 2007; 6 (6): 266-270.

22. Berkovic SF, Andermann F, Shoubridge EA et al. Mitochondrial dysfunction in multiple symmetrical lipomatosis. Ann Neurol 1991; 29 (5): 566-569.

23. Leiva SF, Navachia D, Nigro N, Ibarra R, Cresto JC. Lipoma in the thyroid? J Pediatr Endocrinol Metabol 2004; 17 (7): 1013-1015.

24. Pack S, Turner ML, Zhuang Z et al. Cutaneous tumors in patients with multiple endocrine neoplasia type 1 show allelic deletion of the MEN1 gene. J Invest Dermatol 1998; 110 (4): 438-440.

25. Garfinkel HA. A massive lipoma in a patient receiving chlorpropamide therapy. Postgrad Med J 1971; 47 (544): 137-138.

26. Hurstcad A. Mulder D. MacCarty C. Non-traumatic progression paralysis of the deep radial nerve. Arch Neurol Psychiat 1955; 79: 269-272.

27. Zahrawi F. Acute compression ulnar neuropathy at Guyon's canal resulting from lipoma. J Hand Surg Am 1984; 9 (2): 238-239.

28. Macfarland G, Hoffer M. Paralysis of the intrinsic muscles of the hand secondary to lipoma in Guyon's tunnel. J Bone Joint Surg Am 1971; 53 (2): 375-376.

29. Leffert R. Lipoma of the upper extremity. J Bone Joint Surg 1972; 54 (2): 1262-1266.

30. Aghasi M, Rzctclny V, Axner A. The flexor digitorurn superficialis as a cause of bilateral carpal tunnel syndrome and trigger wrist. J Bone Joint Surg 1980; 62 (2): 134-135.

31. Rodríguez J, Phalen G. Lipoma in the hand and wrist. Cleve Clin Q 1970; 37: 201-205.

32. Paarlberg D, Linscheid R, Soule E. Lipomas of the hand. Mayo Clinic Proc 1972; 47: 121-124.

33. Posch JL. Tumors of the hand. J Bone Joint Surg Am 1956; 38-A (3): 517-539.

34. Flores LP, Carneiro JZ. Peripheral nerve compression secondary to adjacent lipomas. Surg Neurol 2007; 67 (3): 258-262.

35. James JJ, Robin A, Wilson M, Evans AJ. The breast. In: Adam A, Dixon AK, eds. Grainger \& Allison's diagnostic radiology. 5th ed. Philadelphia, Pa: Elsevier 2008.

36. Ant R, Poh AC, Hwang SG. An unusual case of an intramuscular lipoma of the pectoralis major muscle simulating a malignant breast mass. Ann Acad Med Singapore 2005; 34 (3): 275-256. 
37. Dooms GC, Hricak H, Sollitto RA, Higgins CB Lipomatous tumors and tumors with fatty component: MR imaging potential and comparison of MR and CT results. Radiology 1985; 157 (2): 479-483.

38. Egund N, Ekelund L, Sako M, Persson B. CT of softtissue tumors. AJR Am J Roentgenol 1981; 137 (4): 725-729.

39. Halldorsdottir A, Ekelund L, Rydholm A. CT-diagnosis of lipomatous tumors of the soft tissues. Arch Orthop Trauma Surg 1982; 100 (4): 211-216.

40. Chung WJ, Chung HW, Shin MJ et al. MRI to differentiate benign from malignant soft-tissue tumors of the extremities: a simplified systematic imaging approach using depth, size and heterogeneity of signal intensity. Br J Radiol 2012; 85 (1018): e831-e836.

41. Oster LH, Blair WF, Steyers CM. Large lipomas in the deep palmar space. J Hand Surg Am 1989; 14 (4): 700-704.

42. Friedlander HL, Rosenberg NJ, Graubard DJ. Intraneural lipoma of the median nerve. Report of two cases and review of the literature. J Bone Joint Surg Am 1969; 51 (2): 352-362.

43. Choi CW, Kim BJ, Moon SE, Youn SW, Park KC, Huh $\mathrm{CH}$. Treatment of lipomas assisted with tumescent liposuction. J Eur Acad Dermatol Venereol 2007; 21 (2): 243-246.

44. Berger A, TanzellaU. Endoscopically-assisted lipoma removal. Langenbecks Arch Chir Suppl Kongressbd. 1998; 115: 1538-1540.

45. Kopera D, Binder B, Toplak H. Intralesional lipolysis with phosphatidylcholine for the treatment of lipomas: pilot study. Arch Dermatol 2006; 142 (3): 395-396.

46. Rotunda AM, Ablon G, Kolodney MS. Lipomas treated with subcutaneous deoxycholate injections. J Am Acad Dermatol 2005; 53 (6): 973-978.

47. Ergun T, Lakadamyali $H$, Derincek A, Cagla Tarhan $\mathrm{N}$, Ozturk A. Magnetic resonance imaging in the visualization of benign tumors and tumor-like lesions of hand and wrist. Curr Prob Diag Radiol 2010; 39 (1): 1-16.

48. Myhre-Jensen O. A consecutive 7-year series of 1331 benign soft tissue tumors. Clinicopathologic data. Comparison with sarcomas. Acta Orthop Scand 1981; 52 (3): 287-293.

49. Hoglund M, Muren C, Brattstrom G. A statistical model for ultrasound diagnosis of soft-tissue tumors in the hand and forearm. Acta Radiol 1997; 38 (3): 355-358.

Correspondencia:

Dr. Israel Salazar Vizuet

Nicolás San Juan S/N Esq. Ganadería y Parque, Rancho Cuauhtémoc,

Col. Ex Hacienda Magdalena, 50010,

Municipio Toluca, Estado de México.

E-mail: israelsv23@hotmail.com

Conflicto de intereses: Los autores declaran no tener conflicto de intereses. 